Berger, I., Dakwar-Kawar, O., Grossman, E. S., Nahum, M., \& Cohen Kadosh, R. (in press). Scaffolding the Attention-Deficit/Hyperactivity Disorder Brain Using

Transcranial Direct Current and Random Noise Stimulation: a Randomized Controlled

Trial. Clinical Neurophysiology

\title{
Scaffolding the Attention-Deficit/Hyperactivity Disorder Brain Using Transcranial Direct Current and Random Noise Stimulation: a Randomized Controlled Trial
}

Berger, Itai ${ }^{1,2, \#, *}$, Dakwar-Kawar, Ornella ${ }^{3, \#}$, Grossman, Ephraim S. ${ }^{1}$, Nahum, Mor ${ }^{3}$, \& Cohen Kadosh, Roi ${ }^{4, *}$

${ }^{1}$ Pediatric Neurology Unit, Hadassah-Hebrew University Medical Center

${ }^{2}$ School of Social Work and Social Welfare, Hebrew University of Jerusalem

${ }^{3}$ School of Occupational Therapy, Hebrew University of Jerusalem

${ }^{4}$ Wellcome Centre for Integrative Neuroimaging, Department of Experimental Psychology,

University of Oxford

\# Equally contributed

*Correspondence to:

Dr. I. Berger, MD

Assuta Ashdod University Hospital Faculty of Health Sciences, Ben-Gurion University of the Negev

7 Harefua St. Ashdod, Israel

Tel: +972 (0)25852300

Email address: dr.itai.berger@gmail.com
Prof. R. Cohen Kadosh, PhD

University of Oxford

New Radcliffe House

Oxford, OX2 6GG

England

Tel: +44 (0)1865 271385

Email address:

roi.cohenkadosh@psy.ox.ac.uk 


\section{$\underline{\text { Abstract }}$}

Objective: Improving symptomology and cognitive deficits in neurodevelopmental disorders is a crucial challenge. We examined whether neurostimulation protocols, which have been shown to yield long-term effects when combined with cognitive training, could benefit children with Attention-deficit/hyperactivity-disorder (ADHD), the most common neurodevelopmental disorder in childhood.

Methods: We used a randomized double-blind active-controlled crossover study of 19 unmedicated children with ADHD, who received either anodal transcranial direct current stimulation (tDCS) over the left dorsolateral prefrontal cortex (dlPFC) or random noise stimulation (tRNS) over the bilateral dlPFC, while completing executive functions training. Results: For our primary outcome, tRNS yielded a clinical improvement as indicated by the reduced $\mathrm{ADHD}$ rating-scale score from baseline, and in comparison to the changes observed in tDCS. The effect of brain stimulation one week after completion of treatment yielded further improvement, suggesting a neuroplasticity-related effect. Finally, tRNS improved working memory compared to tDCS, and a larger tRNS effect on ADHD rating-scale was predicted for those who showed the greatest improvement in working memory.

Conclusions: We found that our intervention can have a lasting effect, rather than a merely immediate effect as was shown for in previous medical interventions in ADHD.

Significance: Our results provide a promising direction toward a novel intervention in ADHD.

Keywords: ADHD, tRNS, tDCS, intervention, neuroplasticity

\section{Highlights}

- $\quad$ tRNS improved clinical symptoms in unmedicated children with ADHD

- The improvement using tRNS was greater than tDCS with a montage that was highlighted as promising in previous meta-analyses

- The effect of intervention yielded further improvement after completion of treatment, suggesting a neuroplasticity-related effect. 


\section{Introduction}

Attention deficit hyperactivity disorder (ADHD) is the most common neurodevelopmental disorder in childhood, with significant negative lifetime outcomes (Greydanus et al., 2007). Despite proven effects of combinations of pharmacological and psychosocial interventions, there is still a need for improvement of cognitive dysfunction and behavioral symptoms that are only partially covered by current interventions (Moldavsky and Sayal, 2013). These factors highlight the pressing need for novel, efficacious interventions, and transcranial electrical stimulation (tES) has been highlighted as one of possible intervention (Krause and Cohen Kadosh, 2013).

tES involves the application of a weak current (mostly 1-2 mA) to the brain via skinelectrode interface, creating an electric field that modulates neuronal activity (Polania et al., 2018). tES has an excellent safety profile, which makes it an appealing treatment method for children and adolescents (Krishnan et al., 2015). Here we used two types of tES: transcranial direct current stimulation (tDCS) and transcranial random noise stimulation (tRNS). tDCS is the most frequently used form of tES, and it has been suggested that the cortex beneath the anodal electrode typically becomes more excitable whereas the cathodal site has decreased excitability (Polania et al., 2018). The delivery of tRNS uses the same equipment as tDCS to stimulate neuronal activity. However, the mechanisms by which tRNS influences brain activity are different (Chaieb et al., 2015). In addition, in tRNS both electrodes can be used to increase cortical excitability (Terney et al., 2008). Previous studies, mainly in adults, have shown that when several sessions of tDCS or tRNS are applied during cognitive training, the effects can last from weeks to months (e.g., Brevet-Aeby et al., 2019, Reis et al., 2009, Snowball et al., 2013).

One of the most influential theories of the neural basis of ADHD suggests that deficient inhibitory control mechanisms give rise to executive dysfunction, which is likely 
genetically influenced (Sonuga-Barke, 2005). Inhibitory control is processed during the maturation of basal ganglia-thalamo-cortical circuit. Previous studies have shown that ADHD is associated with structural and functional abnormalities within this circuit (Aron et al., 2004, Christakou et al., 2004). An updated meta-analysis suggests that anodal tDCS over the left dlPFC can yield a small-to-medium effect size on neuropsychological deficits, such as inhibition and working memory, in ADHD (Salehinejad et al., 2019). Another recent metaanalysis, which focused on clinical symptoms in ADHD, has suggested that tDCS over the left dlPFC improves inattention and impulsivity (Brauer et al., in press). These results reflects the enthusiasm and promise from tDCS as a potential neurointervention method in ADHD (see also Salehinejad et al., 2020).

To date tRNS has not been used in the case of ADHD, as it is a more novel form of brain stimulation (Polania et al., 2018). However, tRNS in healthy adults successfully improved cognitive functions including attentional control, with stronger effects shown for individuals with poorer attentional control (Harty and Cohen Kadosh, 2019). In a small sample of children with dyscalculia, tRNS over bilateral dlPFC during numerical training has shown positive effects on numerical training compared to sham (placebo) stimulation (Looi et al., 2017). Moreover, accumulated evidence have suggested that tRNS could yield stronger effects than tDCS (Fertonani et al., 2011, Simonsmeier et al., 2018).

The goal of our study was to compare the beneficial effects of tRNS and tDCS when combined with executive function (EF) training in ameliorating symptoms and $\mathrm{EF}$ in unmedicated children with ADHD. Each tES method was applied for 5 consecutive days along with EF training. We limited each arm to 5 days as previous tDCS and tRNS studies on healthy adults have yielded lasting effects with protocols of a similar duration or even shorter (Cappelletti et al., 2013, Reis et al., 2009, Snowball et al., 2013). Endurance of effects was measured one week after the end of the intervention protocol. We expected that both 
stimulation protocols will lead to clinical and cognitive improvement, and our motivation was to examine any potential differential effects between tRNS and tDCS.

\section{$\underline{\text { Methods }}$}

\section{Study Design}

We conducted a randomized double-blind active-controlled crossover study of children diagnosed with ADHD. Twenty-two children were assessed for eligibility, 21 children were recruited for the study, and 19 participants completed it (see Figure 1 for the Consolidated Standards of Reporting Trials (CONSORT) flow diagram). Two participants were excluded from the study: one of them due to complaints of an uncomfortable topical sensation and headaches during the tDCS protocol. The second participant was excluded as the parents reported in the third session behaviour that might meet one of the exclusion criteria (the expression of self-harm thoughts), which was present already two months before study participation but was not reported at screening.

\section{[Insert Figure 1]}

All children were newly diagnosed and drug naïve (Table S1). Given the difficulties in recruiting this population, and for cognitive training that requires longer protocols, we chose in the present study to use a within-subject design. This approach allowed us to control better for individual differences that are impossible to perfectly match in a between-subject design, and at the same time allowing more powerful design, with the given sample size. For example, in a between-subject design the sample size required to detect an effect with $\alpha=.05$, power $(1-\beta)=.8$, and an effect size of Cohen's $d=.68$ using a t-test with a non-directional hypothesis is 72 , more than 3.5 times the sample size required using a within-subject design. 
Following screening, eligible participants were assessed at baseline and then randomized into receiving either tDCS or tRNS first in week 1, along with computerized EF training. Each group received either tDCS or tRNS treatment for 5 consecutive days (one treatment session each day). Following a one-week break, there was a crossover between the groups in week 3: those who received tDCS in the first week received tRNS in the third week, while those who received tRNS in the first week received tDCS in the third week (Figure 2). Parents and children were blinded to treatment method order. This allowed us to compare the different treatment in a within-subject design, as well as to examine one-week post-treatment effects to assess lasting effects. The assessment battery was repeated at the end of each week. The total duration of subject participation in the study was 4 weeks. All study-related activities were conducted in a research lab at the School of Occupational Therapy of the Hebrew University of Jerusalem.

[Insert Figure 2]

\section{Study Population}

The study included children aged 7-12 years old. Participants were recruited (between 03/2018-03/2019) among children referred to the ADHD clinic by paediatricians, general practitioners, teachers, psychologists, or parents. All participants agreed to participate in the study (verbal assent) and their parents gave written informed consent to the study. The authors assert that all procedures contributing to this work comply with the ethical standards of the relevant national and institutional committees on human experimentation and with the Helsinki Declaration of 1975, as revised in 2008. All procedures involving human patients were approved by approved by the Helsinki Committee (IRB) of the Hebrew University and Hadassah Medical Center (Jerusalem, Israel). 
Initially, the local IRB approved a total number of 100 participants for this study. For safety reasons we were asked to summarise the data of the first 20 participants in order to assess safety and tolerability. Upon clinical review by the study team and the IRB - if all safety criteria are met, the study could proceed to recruit another 80 participants. That is the reason we chose to include all 20 participants in the tDCS-tRNS arm, so safety and tolerability, as well as efficacy, will be assessed for both methods and will allow us to revise accordingly the testing plans for the future participants. However, due to the repeated lockdowns and termination of funding we could not continue and recruit the full number. The study is registered at ClinicalTrials.gov (identifier NCT03104972).

A power analysis revealed that the obtained sample size, power $=.8$, and $\alpha=.05$ would allow to detect an effect with an obtained effect size of .68. This is due to the within-subject design, which allows, with the given sample size, for more power to detect an effect compared to previous studies, including a recent trial that used a between-subject design $(n=32$ in one group and $n=30$ in another) and led to Food and Drug Administration (FDA) approval of its use in brain stimulation to treat ADHD (McGough et al., 2019).

Inclusion criteria: Each child scored above the standard clinical cut-off values for ADHD symptoms on ADHD DSM-5 scales (American Psychiatric Association, 2013, DuPaul et al., 2016), and met the criteria for ADHD according to DSM-5, using the "gold standard" procedure as described by the American Academy of Pediatrics, and including a semistructured interview of the patient and parents by a specialist in paediatric neurology and child development, a neurological examination, and ADHD rating scale (ADHD-RS) diagnostic questionnaires (DuPaul et al., 2016).

Exclusion criteria: Children were excluded from the study if they had one of the following: a chronic neurological disease, epilepsy in the participant or in a first-degree relative, intellectual disability, other chronic conditions, chronic use of medications, or other primary 
psychiatric diagnosis (e.g., depression, anxiety, psychosis). The Hebrew translation of the Kiddie-SADS-Lifetime Version (Kaufman et al., 2000) was used to assess axis-I disorders in participants according to DSM-5 criteria (Kaufman et al., 2000).

Prospective resting-state electroencephalography was performed at screening in order to rule out an unknown existence of epileptiform activity. Electroencephalography records were standardized and recorded with g.Recorder software (gTec, Schiedlberg, Austria), using a 64-channel wireless electroencephalography cap system (g.Nautilus) with gel-based electrodes.

\section{Primary Outcome Measure}

The primary outcome measure of the study is the total score of the ADHD-RS diagnostic questionnaire completed by the parents (DuPaul et al., 2016). This scale is of well-accepted validity and reliability, regarded as standards in ADHD diagnosis and treatment effect. The ADHD-RS-5 contains 18 items based on the wording used to describe those items in the DSM-5. The 18 items are presented in the context of a two-factor structure beginning with the nine inattention (IN) symptoms followed by the nine hyperactive-impulsive (HI) symptoms. Parents rate each of these items on a 4-point Likert frequency scale that can be scored 0 (never or rarely), 1 (sometimes), 2 (often), or 3 (very often). IN and HI total symptom severity scores categorically generate IN and HI symptom counts. The symptom count for IN is determined by summing the number of IN items receiving ratings of 2 (often) or 3 (very often). The symptom count for HI is calculated in a similar fashion. Thus, for both IN and HI, symptom counts range from 0 to 9 in accordance with DSM-5 criteria and 18 is the maximal possible scoring for the entire scale (Anastopoulos et al., 2018). Two participants were excluded from this analysis as their parents did not provide the ADHD-RS post-intervention immediately after the intervention and 1 week later.

\section{Secondary outcome measures:}




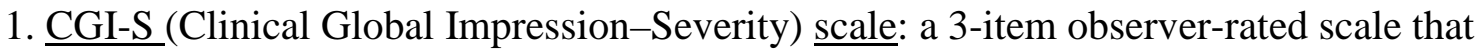
measures illness severity, as assessed by the treating clinician (Guy, 1976). Scoring the CGI$\mathrm{S}$ is rated on a 7-point scale, with the severity of illness scale ranging from 1 (normal) to 7 (severely ill).

2. MOXO-CPT (NeuroTech Solutions Ltd): a standardized computerized test that measures attentional performance (Berger et al., 2017). The MOXO-CPT includes four performance indices: attention, timing, impulsivity, and hyperactivity.

3. Digit Span: a subtest of the Wechsler Intelligence Scale for Children (WISC)-Fourth edition that measures short-term auditory memory and attention (Wechsler, 2003).

\section{Study Interventions}

Participants completed computerized EF training along with either tDCS or tRNS.

\section{Computerized EF Training}

Participants completed training using the ACTIVATE ${ }^{\mathrm{TM}}$ training program, delivered on a tablet (Wexler et al., 2016). This gamified EF training includes different mini-games that target different EF components: working memory, cognitive flexibility, response inhibition, and sustained attention (Wexler et al., 2016). Each training session included 4 mini-games, each played for 5 minutes, which coincided with the tES protocol. The training starts at a basic level and adaptively progresses to move advanced levels, which include more complex tasks, depending on individual performance. While the present cognitive training has been used in previous studies that aimed to improve academic performance in typically developing children (Wexler et al., 2016), and in some preliminary studies in children with ADHD (de Oliveira Rosa et al., 2019), the outcome measures in previous studies never included ADHDRS, which prevented us from estimating the effect size expected from such training alone. As our study focused on comparing the efficacy of two tES methods, a detailed description of 
the EF training protocol is beyond the scope of this paper, but can be found in the Supplementary Information.

\section{$\underline{\text { Transcranial Electrical Stimulation }}$}

Both tDCS and tRNS were applied using semi-dry $5 \mathrm{X} 5 \mathrm{~cm}$ electrodes using the NovoStim device (Tech InnoSphere Eng. Ltd., Haifa). The NovoStim device is a research and investigational device, pending FDA and medical CE approval. Stimulation was delivered for 20 minutes each session, while participants completed the cognitive training (Figure 3). The total stimulation time for each tES protocol was 100 minutes (5 sessions of 20 min each).

$\underline{\text { tDCS. }}$. The current was set to $0.75 \mathrm{~mA}$ based on previous computational modelling of tDCS in children and is estimated to equal that of approximately $1.5 \mathrm{~mA}$ in adults (Kessler et al., 2013). Ramp-up and ramp-down durations were 30 seconds each. These durations were chosen after considering the parameters that would influence current distribution and density at the site of stimulation, such as thinner scalp, less cerebrospinal fluid, and smaller head size of the paediatric population (Kessler et al., 2013). A similar dosage of tDCS was well tolerated by the children and was not associated with adverse effects (Krishnan et al., 2015). The anodal electrode was positioned above the left dIPFC (F3 based on the International 1020 system), while the cathodal electrode was placed over the right supraorbital (Fp2). This montage has been deemed to be the most successful so far based on a meta-analysis of tDCS studies in ADHD (Brauer et al., in press, Salehinejad et al., 2020, Salehinejad et al., 2019, But see for weaker effects in Westwood et al., 2020b).

[Insert Figure 3] 


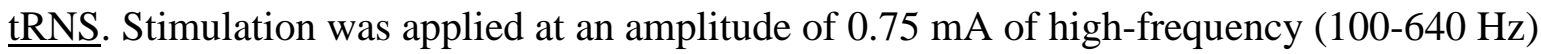
tRNS over the left dlPFC and the right inferior frontal gyrus (IFG), attached under designated electrode positions (F3-F8 based on the International 10-20 system) of the tES cap. These stimulation locations were chosen based on their involvement in executive control and inhibition processes (Aron et al., 2004, Christakou et al., 2004). Ramp-up and ramp-down durations were the same as in the tDCS condition. This montage was chosen due both to the advantage of this neurostimulation polarity-independent method and to its ability to yield excitatory stimulation without parallel inhibitory effects (Terney et al., 2008). Moreover, a similar montage was used in previous tRNS studies in the field of cognitive training in healthy young adults and children with dyscalculia (Looi et al., 2017, Snowball et al., 2013). To mitigate the possibility that the research assistant will notice the differences in the montages between tDCS and tRNS, and would be biased toward a given montage, we alternated three naive research assistants throughout this study.

\section{Statistical Analysis}

To examine treatment effects, we used linear mixed effects models, which account for within-subject correlations more optimally compared to ANOVA and automatically handle missing values, allowing maximum use of available data (Seltman, 2009). We used the Rpackage nlme (Pinheiro et al., 2017) to perform the linear mixed effects analysis with maximized log-likelihood on the outcome measures, and subjects as the random factor. We examined outcomes immediately post-treatment and one week later for each stimulation type, and included stimulation type (tDCS, tRNS) and time (immediately after treatment and one week post-treatment) as predictors. We included baseline performance as a covariate in our model, rather than use a subtraction score (i.e., post-treatment minus baseline). Including baseline performance as a covariate allows for a better adjustment for minor differences in the pre-treatment means. In contrast, subtraction score contains measurement error from both 
the baseline performance and the post-treatment score and is also negatively correlated with baseline performance because of the measurement error (Edwards, 2001, Jamieson, 2007). As we used a within-subjects design, we used the baseline measures at T0 for the first arm, but to allow an accurate assessment of second arm, we recalibrated the participants' baseline measures by using their latest assessment data from $\mathrm{T} 2$ as the new baseline for the second arm (Figure 2).

For all the measures we verified that the residuals were normally distributed using a q-q plot and the Shapiro-Wilk normality test. The only exception was the MOXO-CPT residuals, which were not normally distributed; we therefore applied the Tukey ladder of powers transformation, which is recommended in this case (Tukey, 1977). We also tested for the inclusion of an interaction term in our analysis. In our primary outcome, ADHD-RS, the interaction between stimulation type and time was not significant $[\beta=.14, \mathrm{SE}=.18, \mathrm{t}(35)=.78$, $\mathrm{p}=.44,95 \%$ confidence intervals $(\mathrm{CI})(-.21, .5)]$. A model comparison showed no benefit from a more complex model, favouring the more parsimonious model, which included the main effects of stimulation and time (chi-squared test $=.66, \mathrm{p}=.41$ ). We therefore report this parsimonious model also for the secondary outcome measures. However, as with the other measures, the inclusion of the interaction term between stimulation and time was not significant. We also explored the effect of order (tRNS first followed by tDCS, vice versa), but this variable was not significant $[\beta=-.12, \mathrm{SE}=.12, \mathrm{t}(34)=-1.04, \mathrm{p}=.3,95 \% \mathrm{CI}(-.37, .11)]$, and a model comparison preferred the simpler model that did not include this variable (chisquared test $=1.12, \mathrm{p}=0.29)$.

\section{Data availability}

Data is available upon reasonable request from the first author. 


\section{$\underline{\text { Results }}$}

\section{Side Effects and Safety Issues}

There were 61 records of side effects reported, none of which were considered clinically significant (Table S2).

\section{Primary Outcome Measure}

For the primary outcome, we measured the ADHD-RS total score post-treatment immediately after the intervention (tRNS/tDCS) and one week later, while covarying for the baseline score. The analysis revealed a main effect of stimulation type, indicating greater improvement for tRNS than for $\mathrm{tDCS}[\beta=-.42(\mathrm{SE}=.18), \mathrm{B}=-1.98(\mathrm{SE}=.87), \mathrm{t}(35)=-2.28$, $\mathrm{p}=.028,95 \%$ CI (-3.67, -.29)] Table 1, Figures S1-2). The main effect of time, i.e., immediately after the end of the intervention to one week later, showed a further improvement one week after the end of the treatment $[\beta=-.19(\mathrm{SE}=.09), \mathrm{B}=-1.78(\mathrm{SE}=.86)$, $\mathrm{t}(35)=-2.07, \mathrm{p}=.045), 95 \% \mathrm{CI}(-3.46,-.1)]$. In terms of improvement from baseline, tRNS yielded a mean improvement of 3.47 points $[\mathrm{SE}=1.03, \mathrm{t}(15)=3.35, \mathrm{p}=.004,95 \% \mathrm{CI}(1.31$, 5.64)], while tDCS yielded a mean improvement of .57 points $[\mathrm{SE}=1.19, \mathrm{t}(15)=.47, \mathrm{p}=.64$, 95\% CI $(-1.92,3.06)]$.

\section{[Insert Table 1]}

\section{Changes in Secondary Outcome Measures}

The secondary outcome measures were considered more exploratory. As such, we present them below without applying a correction for multiple comparisons, yet highlight that none of the results was significant at a $\alpha \leq .05$ after applying Bonferroni correction for multiple comparisons.

Changes in Attentional Performance 
The results for the MOXO-CPT subscales and CGI-S (see Tables S3-S7) did not show a significant post-treatment effect of stimulation type (all ps>0.44), aside from the MOXO timing index, which showed larger changes following tRNS compared with those seen following $\mathrm{tDCS}[\mathrm{B}=1.92(\mathrm{SE}=.91), \mathrm{t}(47)=2.11, \mathrm{p}=.04,95 \% \mathrm{CI}(.14,3.7)]$.

Changes in Working Memory and in Short-Term Memory

Performance on the digit span subscale of the WISC (total score of forward and backward span) after the intervention showed a significant effect of stimulation, favouring tRNS over tDCS $[\beta=.34(\mathrm{SE}=.14), \mathrm{B}=1.07(\mathrm{SE}=.44), \mathrm{t}(50)=2.44, \mathrm{p}=.018,95 \% \mathrm{CI}(.22,1.92)]$.

Further analysis indicates that tRNS led to a significantly better performance in the backward digit span only, compared to tDCS [Table 2, backward digit span: $\beta=.33$ (SE=.16), $\mathrm{B}=.63(\mathrm{SE}=.3), \mathrm{t}(51)=2.12, \mathrm{p}=.038,95 \% \mathrm{CI}(.04,1.22)$; forward digit span: $\beta=.04(\mathrm{SE}=.16)$, $\mathrm{B}=.058(\mathrm{SE}=.24), \mathrm{t}(51)=.24, \mathrm{p}=.81,95 \% \mathrm{CI}(-.41, .52)$, Table S8].

[Insert Table 2]

\section{Examining the Link between Clinical and Cognitive Changes}

Next, we examined whether the improvement in the ADHD-RS score under the tRNS protocol depends on the changes in working memory (WM) performance (the backward digit span score). We ran a moderation analysis and predicted the post-treatment ADHD-RS score by stimulation type and the post-treatment backward digit span score, while controlling for the ADHD-RS and backward digit span scores at baseline. This analysis revealed a trend toward a significant interaction between stimulation type and the post-treatment backward digit $\operatorname{span}$ score $[\beta=-.41(\mathrm{SE}=.23), \mathrm{B}=-1.01(\mathrm{SE}=.56), \mathrm{t}(53)=-1.81, \mathrm{p}=.075,95 \% \mathrm{CI}(-2.09$, 0.06), Table S9 and Figure S3]. A simple slopes analysis revealed that this trend stemmed from a significant improvement in ADHD-RS for tRNS vs. tDCS in those who had showed 
Scaffolding the ADHD Brain

the largest improvement in the backward digit span test $[\beta=-.62(\mathrm{SE}=.29), \mathrm{B}=-2.91$

$(\mathrm{SE}=1.36), \mathrm{t}(53)=-2.14, \mathrm{p}=.037), 95 \% \mathrm{CI}(-.24,-5.57)]$.

\section{$\underline{\text { Discussion }}$}

The most notable results in our study are the improvements on ADHD-RS scores following tRNS and EF training relative to baseline and to tDCS and EF training. These promising results on the tRNS protocol support those of several studies in healthy young adults (BrevetAeby et al., 2019, Snowball et al., 2013, Terney et al., 2008). Importantly, the results showed a further significant improvement 7 days after the end of the treatment, mirroring a similar lasting tRNS effect in previous studies on healthy adults (Brevet-Aeby et al., 2019, Snowball et al., 2013).

Our results are further supported by a tRNS effect on the MOXO timing index score, which reflects cognitive processing speed, i.e., the speed at which a person is able to perceive and react to stimuli in the environment (Nielsen et al., 2017). More importantly, we observed a tRNS effect on the backward digit span test, which measures WM capacity. This last effect is expected given that our cognitive training targeted WM as one of the EFs that have been shown to be impaired in children with ADHD (Barkley, 1997). Moreover, a greater improvement of ADHD-RS by tRNS was predicted by a greater improvement in the backward digit span test from baseline. However, the interaction between brain stimulation and WM in predicting ADHD-RS was only marginally significant.

\section{Potential Mechanisms}

The most prevalent explanation for tRNS is stochastic resonance (Fertonani and Miniussi, 2017, Pavan et al., 2019, Terney et al., 2008, van der Groen and Wenderoth, 2016). Stochastic resonance describes the phenomenon of introducing an appropriate level of random noise to enhance the output of subthreshold signals. With respect to tRNS, it suggests 
that the application of weak electric currents amounts to an introduction of neural noise, which improve information processing at the neuronal level (Terney et al., 2008). According to this framework the effect of tRNS in the present study might be attributed to amplifying underactive basal ganglia-thalamo-cortical circuits that has been associated with ADHD (Aron et al., 2004, Christakou et al., 2004). In this scenario, targeting the prefrontal cortex impact the dorsal neo-striatum via excitatory glutaminergic cells, the basal ganglia to the dorsomedial thalamus via inhibitory projections, and the thalamus back to the prefrontal cortex via excitatory projections (Castellanos et al., 2002).

A second mechanistic explanation for the tRNS in the present study is coming from a combined electroencephalography-tRNS study that found that the behavioural improvements of tRNS above the dIPFC vs. sham tRNS are associated with alterations in amplitude of attention and preparatory markers. Those results suggest that the enhancement effect of tRNS when applied above the dIPFC acts by effecting general attentional mechanisms during cognitive training (Sheffield et al., 2020). However, it is important to highlight that the abovementioned study included healthy young adults.

However, another possibility is that tRNS impacted oscillations between 140-220 Hz ("ripples"), which are involved in learning and long-term potentiation (Jadhav et al., 2016). Ripple oscillations underlie learning via Hebbian requirements for synaptic modification, and are attributed to the hippocampus as well as the prefrontal cortex (Jadhav et al., 2016). If tRNS modulated ripple oscillations and by that causally alter learning and long-term potentiation in ADHD then a more optimal tRNS frequencies would be within 140-220 Hz, rather than $100-640 \mathrm{~Hz}$ as in this study. Namely, while in the non-cognitive domain highfrequency tRNS $(101-640 \mathrm{~Hz})$ has been suggested to be more beneficial than low-frequency $(0.1-100 \mathrm{~Hz})$ (Terney et al., 2008), the prediction of the ripple oscillations hypothesis is that 
the most optimal parameters will appear in the range of 140-220 Hz (Jadhav et al., 2016). It might be that transient tRNS effect, as was found in other studies, might be due to other mechanisms such as stochastic resonance, while the neuroplasticity effects could be due to ripple oscillations.

In addition, a recent study in mice that aimed to progress the understanding of tRNS effect on the developing brain has revealed that identical tRNS current density and duration per day over multiple sessions (in this case 9 sessions, twice a week) over the prefrontal cortex has yielded changes in reduction in glutamic acid decarboxylase (GAD) 65/67 but not vesicular glutamate transporter 1 . Such effect was maximal in the location beneath the electrode but not in a deeper location (Sanchez-Leon et al., in press). Such findings strengthening our suggestion that tRNS impacts neuroplastic mechanisms, and at least in mice involves the GABAergic system.

While describing the potential mechanisms for tRNS, an existing question is why tRNS was better than tDCS in the present study. While we do not have the needed data to shed light on this question, in addition to the potential mechanisms that tRNS might have played in our observed results, we would like to suggest a couple of potential reasons. First, tRNS allowed us to use two electrodes that have shown to have excitatory effect, rather than one excitatory and one inhibitory effect in tDCS. It is unclear if the additional excitatory effect of the tRNS over the right IFG and/or the cathodal effect of tDCS over the supraorbital have contributed to the increased efficacy of tRNS. Second, tRNS provide alternating current in a wide range of frequencies. Such feature could have allowed more variability in terms of the stimulation protocol, compared to the fixed direct current in tDCS and therefore impacting more individuals despite their assumed heterogeneity. Similarly, tRNS is less sensitive to cortical folding than tDCS (Terney et al., 2008), and therefore reducing the impact of anatomical variations between participants. Third, by inducing random noise at the 
neuronal level, tRNS may increase uncertainty, which is associated with greater level of plasticity (Chang and Merzenich, 2003, Frankenhuis and Fraley, 2017). This effect might allow greater opportunity for the central nervous system to change. While such effect of increased uncertainty might be also induced by tDCS, the effect of tRNS might be greater due to its greater impact on inducing neuronal noise. To our knowledge, this idea is novel and has not been yet examined in the field of brain stimulation.

Note, that the aim of the current study was to improve clinical symptoms in ADHD by combining theoretically-motivated EF cognitive training with the currently most promising transcranial electrical stimulation protocols. tDCS and tRNS have been shown to work, at least partially, on different mechanisms (Antal and Herrmann, 2016, Chaieb et al., 2015, Liebetanz et al., 2002, Nitsche et al., 2004). We therefore did not match either of the protocols with regard to different factors such as electrode positions. In this respect, our approach is very similar to the approach that is adopted in pharmacological studies, where not the dose of different drugs are matched, but the best dose for each drug with maximal effects on brain and behavior is investigated (Chamberlain et al., 2006). As such, our goal was to test the efficacy of tRNS and tDCS protocols using the parameters that yielded promising results in previous studies. Therefore, instead of equating the tRNS and tDCS protocols (if possible at all) and comparing the involved mechanisms, which would be an interesting and challenging question per se, our motivation was different; to yield the best clinical results. In this respect, future studies should examine whether further optimizing tDCS and tRNS protocols using different parameters could yield different conclusions, and whether the present beneficial effect of tRNS is limited to the developing brain as well as other ADHD characteristics (Lipka et al., Submitted). However, few studies that have found greater benefit of tRNS vs. tDCS in adults may suggest that its advantage may not be necessarily restricted 
to the developing brain (Brem et al., 2018, Fertonani et al., 2011, Ghin et al., 2018, Inukai et al., 2016).

\section{Comparing the Present Approach to Previous Studies}

There are a few differences that are worth emphasizing when comparing our approach to the recent promising findings on trigeminal nerve stimulation as a treatment for ADHD (McGough et al., 2019). Our results are based on lower stimulation intensity (.75 mA vs. 2-4 $\mathrm{mA}$ ) and shorter treatment duration (100min vs. 13,440min in total), and they show persistent and even increasing improvement after treatment, indicating plasticity-related effects. This is in contrast to the short-lived immediate improvement and significant deterioration one week after the end of the treatment associated with trigeminal nerve stimulation (McGough et al., 2019). Moreover, the estimated effect size in our study on ADHD-RS is higher than the one reported in (McGough et al., 2019) (estimated Cohen's d=.82 on an 18 points scale, and .75 on a 54 points scale vs. .51). This difference is less likely to be due to an inflated effect size due to an underpowered design as the experimental design in our study was more suitable for detecting the observed effect size. The sample size in the present study is the upper range of the sample size used in paediatric ADHD neurostimulation studies (with n=9-21, Salehinejad et al., 2019). Notably, most of these studies did not require the patients and their guardians to come to the lab multiple time as in the present study, which increase difficulties in terms of recruitment, and parental and child's commitment. Moreover, all the children in our study were newly diagnosed and drug naïve.

\section{Potential Limitations and Future Directions}

While we stressed the strength of our approach to ensure balance, we would like to discuss also possible limitations. 
One potential caveat in the present study is the lasting effect of a given intervention (e.g., tRNS). To take this into account we "rescaled" in our statistical model the baseline performance before the beginning of the second intervention, and also examine how the factor order, which was not significant, could influence the results.

In addition, in the present study we chose to compare the effect of tRNS vs. tDCS, rather than sham stimulation. In our view, such an approach is more rigorous as it compares the effect of two stimulation protocols that at the theoretical and the empirical level had an a priori likelihood of leading to successful treatment. Therefore, the obtained results are expected to be stronger when compared to sham stimulation. However, a potential criticism is that tDCS, in contrast to sham stimulation, might yield impairment, rather than improvement. While a future study that includes a sham group is needed to exclude this possibility with great confidence, the criticism is likely unfounded given the accumulated evidence that suggests a beneficial effect of tDCS on ADHD clinical symptoms and neuropsychological deficits under the montage we used (Brauer et al., in press, Salehinejad et al., 2020, Salehinejad et al., 2019). However, this potential concern cannot be excluded entirely given that studies that used tDCS with anodal electrode over the right inferior frontal gyrus has found a detrimental effect compared to sham in adolescents with ADHD (Breitling et al., 2020, Westwood et al., 2020a).

Our present results offer a further motivation to examine the results vs. sham group to strength the confidence in the present results and replicate them, as well as examining the potential benefit of tRNS in a large sample size as a function of ADHD subtype. In addition, compared to the mechanisms involved in other brain stimulation methods, such as tDCS, the neurocognitive mechanisms in tRNS are less known. While we discuss potential mechanistic explanation to our results, further work in humans and animals could shed further light on the 
involved mechanisms, and how tRNS can ameliorate ADHD symptoms, and potentially other clinical conditions.

Acknowledgements. We thank Prof. Katya Rubia for initiating the idea to use the ACTIVATE $^{\mathrm{TM}}$ training program with brain stimulation. We also thank Snir Barzilay, Noam Galon, Yehudit Fox, Romy Goldfus and Noa Ariely for their help with data collection, organization and psychological evaluations. The manuscript has been posted on a preprint server (medRxiv). This research was funded by a grant from the Israel Innovation Authority to Tech Innosphere Engineering Ltd. ODK has been partially supported by a Golda Meir award of the Israeli Ministry of Science and Technology, granted to advanced graduate and postgraduate students in science and technology.

Declarations of Interest: IB serves on the advisory board of Tech InnoSphere Engineering Ltd. RCK serves on the scientific advisory boards of Neuroelectrics Inc. and Tech InnoSphere Engineering Ltd. RCK filed a UK Patent via the University of Oxford for "method for obtaining personalized parameters for transcranial stimulation, transcranial system, method of applying transcranial stimulation". All the other authors reported no biomedical financial interests or potential conflicts of interest.

\section{$\underline{\text { References }}$}

American Psychiatric Association. Diagnostic and statistical manual of mental disorders (DSM-5®): American Psychiatric Pub, 2013.

Anastopoulos AD, Beal KK, Reid RJ, Reid R, Power TJ, DuPaul GJ. Impact of child and informant gender on parent and teacher ratings of attention-deficit/hyperactivity disorder. Psychol Assess 2018;30(10):1390-4.

Antal A, Herrmann CS. Transcranial Alternating Current and Random Noise Stimulation: Possible Mechanisms. Neural Plast 2016;2016:3616807.

Aron AR, Robbins TW, Poldrack RA. Inhibition and the right inferior frontal cortex. Trends Cogn Sci 2004;8:170-7.

Barkley RA. Behavioral inhibition, sustained attention, and executive functions: constructing a unifying theory of ADHD. Psychol Bull 1997;121(1):65.

Berger I, Slobodin O, Cassuto H. Usefulness and Validity of Continuous Performance Tests in the Diagnosis of Attention-Deficit Hyperactivity Disorder Children. Arch Clin Neuropsychol 2017;32(1):81-93.

Brauer H, Breitling-Ziegler C, Moliadze V, Galling B, Prehn-Kristensen A. Transcranial direct current stimulation in ADHD - a meta-analysis of clinical efficacy outcomes. Prog Brain Res in press.

Breitling C, Zaehle T, Dannhauer M, Tegelbeckers J, Flechtner H-H, Krauel K. Comparison between conventional and HD-tDCS of the right inferior frontal gyrus in children and adolescents with ADHD. Clin Neurophysiol 2020;131(5):1146-54.

Brem AK, Almquist JN, Mansfield K, Plessow F, Sella F, Santarnecchi E, et al. Modulating fluid intelligence performance through combined cognitive training and brain stimulation. Neuropsychologia 2018;118(Pt A):107-14. 
Brevet-Aeby C, Mondino M, Poulet E, Brunelin J. Three repeated sessions of transcranial random noise stimulation (tRNS) leads to long-term effects on reaction time in the Go/No Go task. Neurophysiol Clin 2019;49(1):27-32.

Cappelletti M, Gessaroli E, Hithersay R, Mitolo M, Didino D, Kanai R, et al. Transfer of Cognitive Training across Magnitude Dimensions Achieved with Concurrent Brain Stimulation of the Parietal Lobe. J Neurosci 2013;33(37):14899-907.

Castellanos FX, Lee PP, Sharp W, Jeffries NO, Greenstein DK, Clasen LS, et al.

Developmental trajectories of brain volume abnormalities in children and adolescents with attention-deficit/hyperactivity disorder. JAMA 2002;288(14):1740-8.

Chaieb L, Antal A, Paulus W. Transcranial Random Noise Stimulation-induced plasticity is NMDA-receptor independent but sodium-channel blocker and benzodiazepines sensitive. Front Neurosci 2015;9.

Chamberlain SR, Müller U, Blackwell AD, Clark L, Robbins TW, Sahakian BJ. Neurochemical Modulation of Response Inhibition and Probabilistic Learning in Humans. Science 2006;311(5762):861-3.

Chang EF, Merzenich MM. Environmental Noise Retards Auditory Cortical Development. Science 2003;300(5618):498-502.

Christakou A, Robbins TW, Everitt BJ. Prefrontal cortical-ventral striatal interactions involved in affective modulation of attentional performance: implications for corticostriatal circuit function. J Neurosci 2004;24(4):773-80.

de Oliveira Rosa V, Rosa Franco A, Abrahao Salum Junior G, Moreira-Maia CR, Wagner F, Simioni A, et al. Effects of computerized cognitive training as add-on treatment to stimulants in ADHD: a pilot fMRI study. Brain Imaging Behav 2019;14(5):1933-44.

DuPaul GJ, Reid R, Anastopoulos AD, Lambert MC, Watkins MW, Power TJ. Parent and teacher ratings of attention-deficit/hyperactivity disorder symptoms: Factor structure and normative data. Psychol Assess 2016;28(2):214.

Edwards JR. Ten Difference Score Myths. Organizational Res Methods 2001;4(3):265-87.

Fertonani A, Miniussi C. Transcranial Electrical Stimulation: What We Know and Do Not Know About Mechanisms. Neuroscientist 2017;23(2):109-23.

Fertonani A, Pirulli C, Miniussi C. Random Noise Stimulation Improves Neuroplasticity in Perceptual Learning. J Neurosci 2011;31(43):15416-23.

Frankenhuis WE, Fraley RC. What do evolutionary models teach us about sensitive periods in psychological development? Eur Psychologist 2017;22:141-50.

Ghin F, Pavan A, Contillo A, Mather G. The effects of high-frequency transcranial random noise stimulation (hf-tRNS) on global motion processing: An equivalent noise approach. Brain Stimul 2018;11(6):1263-75.

Greydanus DE, Pratt HD, Patel DR. Attention deficit hyperactivity disorder across the lifespan: the child, adolescent, and adult. Dis Mon 2007;53(2):70-131.

Guy W. ECDEU Assessment Manual of Psychopharmacology. Rockville, MD: National Institute of Mental Health, US Department of Health, Education, and Welfare. Psychopharmacol Res Branch 1976:76-338.

Harty S, Cohen Kadosh R. Suboptimal Engagement of High-Level Cortical Regions Predicts Random-Noise-Related Gains in Sustained Attention. Psychol Sci 2019;30(9):131832.

Inukai Y, Saito K, Sasaki R, Tsuiki S, Miyaguchi S, Kojima S, et al. Comparison of Three Non-Invasive Transcranial Electrical Stimulation Methods for Increasing Cortical Excitability. Front Hum Neurosci 2016;10:668.

Jadhav SP, Rothschild G, Roumis DK, Frank LM. Coordinated Excitation and Inhibition of Prefrontal Ensembles during Awake Hippocampal Sharp-Wave Ripple Events. Neuron 2016;90(1):113-27. 
Jamieson J. Difference score. In: Salkind NJ, editor. Encyclopedia of Measurement and Statistics. Thousand Oaks, California: Sage Publications, Inc.; 2007. p. 259-60.

Kaufman J, Birmaher B, Brent DA, Ryan ND, Rao U. K-Sads-Pl. J Am Acad Child Adolesc Psychiatry 2000;39(10):1208.

Kessler SK, Minhas P, Woods AJ, Rosen A, Gorman C, Bikson M. Dosage considerations for transcranial direct current stimulation in children: a computational modeling study. PLoS One 2013;8(9):e76112.

Krause B, Cohen Kadosh R. Can transcranial electrical stimulation improve learning difficulties in atypical brain development? A future possibility for cognitive training. Dev Cogn Neurosci 2013;6(0):176-94.

Krishnan C, Santos L, Peterson MD, Ehinger M. Safety of Noninvasive Brain Stimulation in Children and Adolescents. Brain Stimul 2015;8(1):76-87.

Liebetanz D, Nitsche M, Tergau F, Paulus W. Pharmacological approach to the mechanisms of transcranial DC-stimulation-induced after-effects of human motor cortex excitability. Brain 2002;125:2238 - 47.

Lipka R, Ahlers E-C, Reed TL, Karstens MI, Nguyen V, Bajbouj M, et al. Resolving Heterogeneity in Transcranial Electrical Stimulation Efficacy for Attention Deficit Hyperactivity Disorder. Submitted.

Looi CY, Lim J, Sella F, Lolliot S, Duta M, Avramenko AA, et al. Transcranial random noise stimulation and cognitive training to improve learning and cognition of the atypically developing brain: A pilot study. Sci Rep 2017;7(1):4633.

McGough JJ, Sturm A, Cowen J, Tung K, Salgari GC, Leuchter AF, et al. Double-Blind, Sham-Controlled, Pilot Study of Trigeminal Nerve Stimulation for AttentionDeficit/Hyperactivity Disorder. J Am Acad Child Adolesc Psychiatry 2019;58(4):40311.e3.

Moldavsky M, Sayal K. Knowledge and attitudes about attention-deficit/hyperactivity disorder (ADHD) and its treatment: the views of children, adolescents, parents, teachers and healthcare professionals. Curr Psychiatry Rep 2013;15(8):377.

Nielsen NP, Wiig EH, Bäck S, Gustafsson J. Processing speed can monitor stimulantmedication effects in adults with attention deficit disorder with hyperactivity. Nordic J Psychiatry 2017;71(4):296-303.

Nitsche M, Liebetanz D, Schilitterlau A, Henschke U, Fricke K, Frommann K, et al. GABAergic modulation of DC stimulation-induced motor cortex excitability shifts in humans. Eur J Neurosci 2004;19:2720-6.

Pavan A, Ghin F, Contillo A, Milesi C, Campana G, Mather G. Modulatory mechanisms underlying high-frequency transcranial random noise stimulation (hf-tRNS): A combined stochastic resonance and equivalent noise approach. Brain Stimul 2019;12(4):967-77.

Pinheiro J, Bates D, DebRoy S, Sarkar D, Team RC. nlme: Linear and Nonlinear Mixed Effects Models. R package version 3.1-130, https://CRAN.Rproject.org/package=nlme. 2017.

Polania R, Nitsche MA, Ruff CC. Studying and modifying brain function with non-invasive brain stimulation. Nat Neurosci 2018;21(2):174-87.

Reis J, Schambra HM, Cohen LG, Buch ER, Fritsch B, Zarahn E, et al. Noninvasive cortical stimulation enhances motor skill acquisition over multiple days through an effect on consolidation. Proc Natl Acad Sci 2009;106(5):1590-5.

Salehinejad MA, Nejati V, Mosayebi-Samani M, Mohammadi A, Wischnewski M, Kuo M-F, et al. Transcranial Direct Current Stimulation in ADHD: A Systematic Review of Efficacy, Safety, and Protocol-induced Electrical Field Modeling Results. Neurosci Bull 2020;36(10):1191-212. 
Salehinejad MA, Wischnewski M, Nejati V, Vicario CM, Nitsche MA. Transcranial direct current stimulation in attention-deficit hyperactivity disorder: A meta-analysis of neuropsychological deficits. PLOS ONE 2019;14(4):e0215095.

Sanchez-Leon CA, Sanchez-Lopez A, Gomez-Climent MA, Cordones I, Cohen Kadosh R, Marquez-Ruiz J. Impact of chronic transcranial Random-Noise Stimulation (tRNS) on prefrontal cortex excitation-inhibition balance in juvenile mice. bioRxiv 2020:2020.09.04.282889.

Seltman HJ. Mixed models. A flexible approach to correlated data. In: Experimental design and analysis. Pittsburgh, PA: Carnegie Mellon University, 2009.

Sheffield JG, Raz G, Sella F, Cohen Kadosh R. How can noise alter neurophysiology in order to improve human behaviour? A combined transcranial random noise stimulation and electroencephalography study. bioRxiv 2020:2020.01.09.900118.

Simonsmeier BA, Grabner RH, Hein J, Krenz U, Schneider M. Electrical brain stimulation (tES) improves learning more than performance: A meta-analysis. Neurosci Biobehav Rev 2018;84:171-81.

Snowball A, Tachtsidis I, Popescu T, Thompson J, Delazer M, Zamarian L, et al. Long-Term Enhancement of Brain Function and Cognition Using Cognitive Training and Brain Stimulation. Cur Biol 2013;23(11):987-92.

Sonuga-Barke EJ. Causal models of attention-deficit/hyperactivity disorder: from common simple deficits to multiple developmental pathways. Biol Psychiatry 2005;57(11):1231-8.

Terney D, Chaieb L, Moliadze V, Antal A, Paulus W. Increasing human brain excitability by transcranial high-frequency random noise stimulation. J Neurosci 2008;28:14147-55.

Tukey JW. Exploratory Data Analysis. Reading, MA: Addison-Wesley, 1977.

van der Groen O, Wenderoth N. Transcranial Random Noise Stimulation of Visual Cortex: Stochastic Resonance Enhances Central Mechanisms of Perception. J Neurosci 2016;36(19):5289-98.

Wechsler D. Wechsler intelligence scale for children-Fourth Edition (WISC-IV). San Antonio, TX: The Psychological Corporation 2003.

Westwood SJ, Criaud M, Lam S-L, Lukito S, Wallace-Hanlon S, Kowalczyk OS, et al. Transcranial direct current stimulation (tDCS) combined with cognitive training in adolescent boys with ADHD: a double-blind, randomised, sham-controlled trial. medRxiv 2020a:2020.12.07.20245456.

Westwood SJ, Radua J, Rubia K. Noninvasive brain stimulation in children and adults with attention-deficit/hyperactivity disorder: a systematic review and meta-analysis. J Psychiatry Neurosci 2020b;45(6):190179.

Wexler BE, Iseli M, Leon S, Zaggle W, Rush C, Goodman A, et al. Cognitive Priming and Cognitive Training: Immediate and Far Transfer to Academic Skills in Children. Sci Rep 2016;6:32859. 


\section{Figure Captions}

Figure 1. Consolidated Standards of Reporting Trials (CONSORT) flow diagram of the progress through the phases of the randomized crossover study of the two groups.

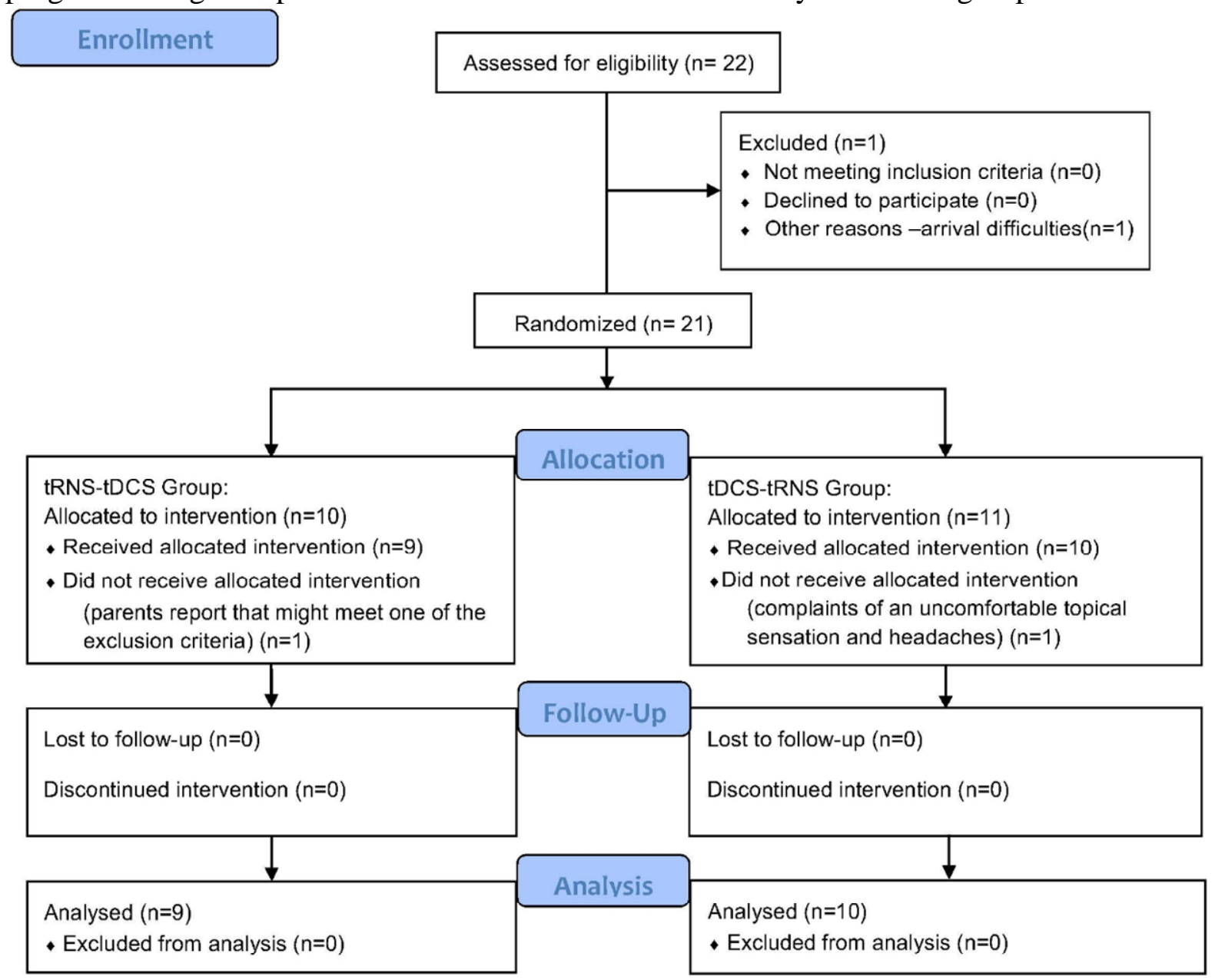


Figure 2. Study Design. Eligible participants with Attention-deficit/hyperactivity-disorder (ADHD) were randomized (Rand) into one of two treatment groups. Baseline measures were acquired (T0) before the randomization into one of the two groups. Participants in both groups received 5 daily treatment sessions in Week 1 and were assessed at the end of this week (T1). At Week 2 no treatment was given and the lasting effect from week 1 was measured (T2). On Week 3 the participants received the treatment that the other group received in Week 1. That is, if participants received transcranial random noise stimulation and executive functions training (tRNS+EF) on Week 1, they received transcranial direct current stimulation and executive functions training (tDCS+EF) on Week 3. To allow an accurate assessment of the new treatment on Week 3, we recalibrated the participants' baseline measures by using their latest assessment data from T2. This new baseline measure was called New T0 (N-T0). We reassessed the participants at the end of Week 3 (N-T1). At Week 4 no treatment was given and the lasting effect from week 3 was measured (N-T2).

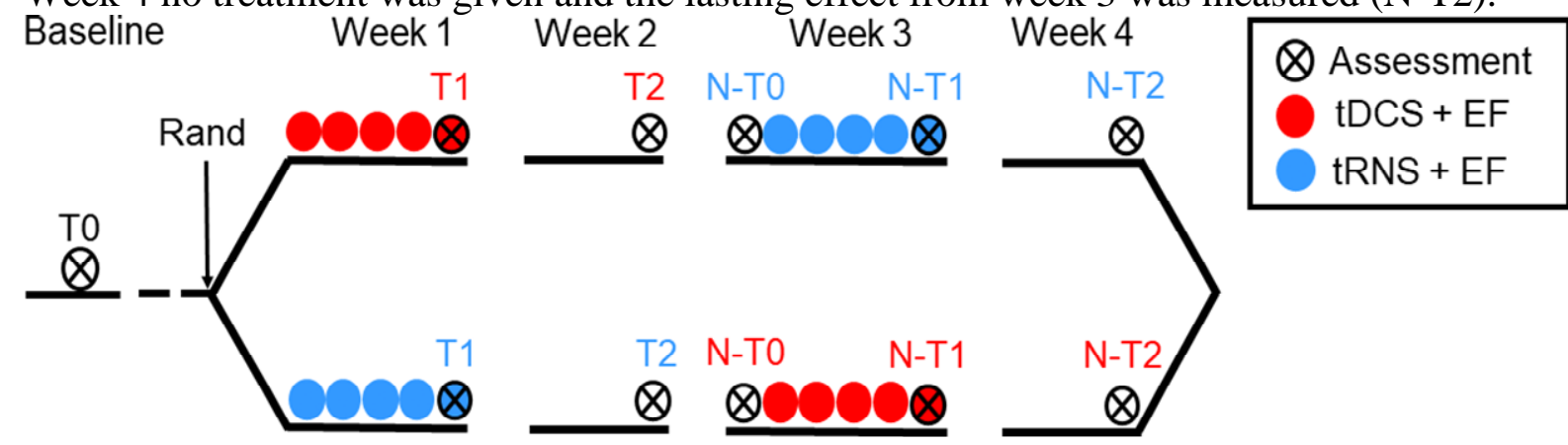


Figure 3. A treatment session of transcranial electrical stimulation combined with executive functions (EF) training. Participants completed 20 minutes of EF training while transcranial random noise stimulation or transcranial direct current stimulation was delivered to them during this period.

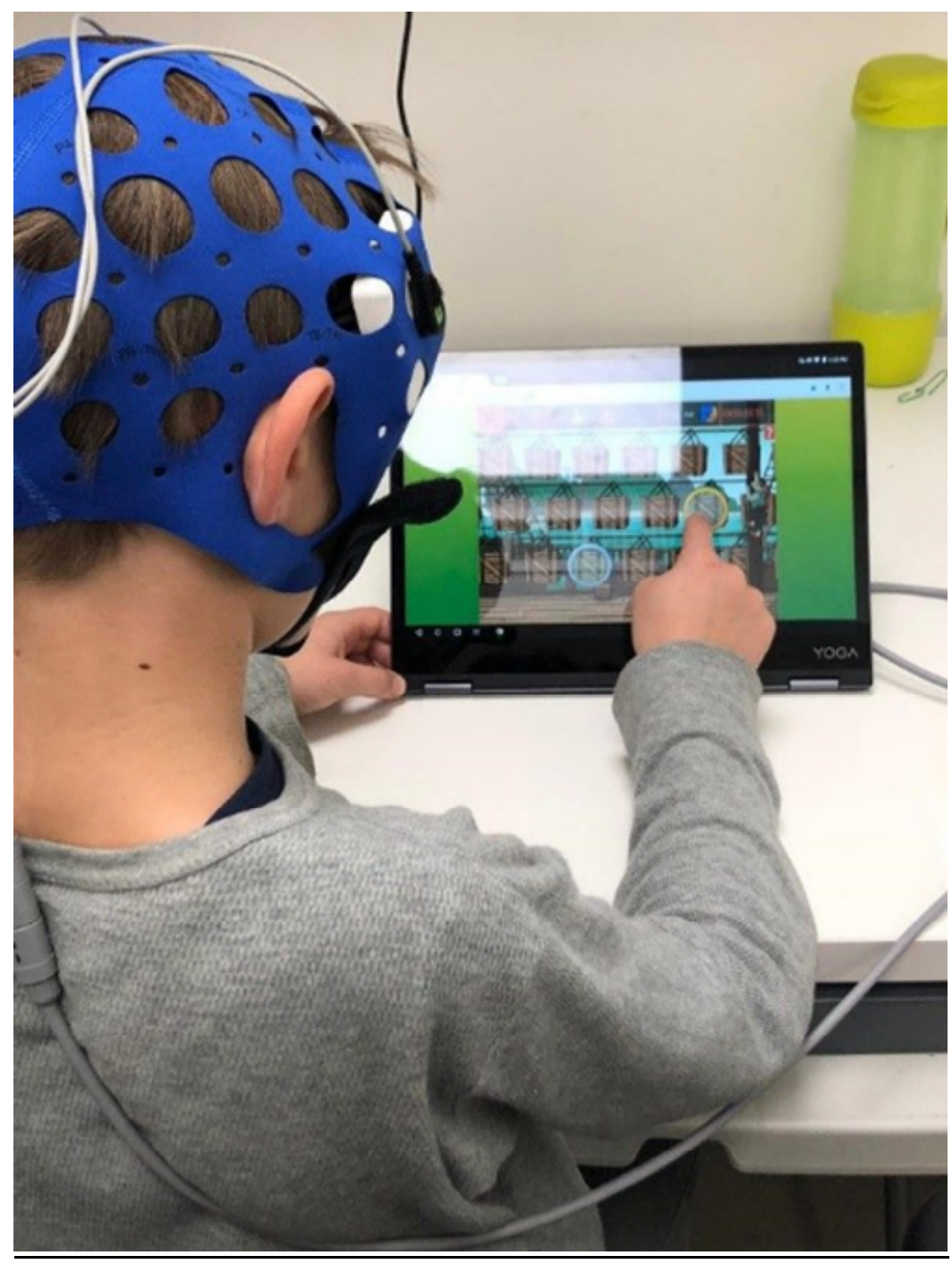




\section{Tables}

Table 1. Beta Weights (Standardized) of the Regression Model with Post-treatment Attention-deficit/hyperactivity-disorder rating scale (ADHD-RS) Score as the Outcome Measure. The results indicate a significant effect for stimulation due to greater reduction in the ADHD-RS score for transcranial random noise stimulation in comparison to transcranial direct current stimulation, and greater improvement, as opposed to deterioration, as time passed following the treatment (one week later). $\mathrm{Std}=$ standard; $\mathrm{DF}=$ degrees of freedom

\begin{tabular}{lccccc} 
& $\beta$ & Std Error & DF & t-value & p-value \\
\hline Intercept & -0.08 & 0.173 & 35 & -0.484 & 0.631 \\
ADHD-RS (baseline) & 0.228 & 0.105 & 35 & 2.166 & 0.037 \\
Stimulation & -0.422 & 0.185 & 35 & -2.285 & 0.028 \\
Time & -0.192 & 0.092 & 35 & -2.075 & 0.045 \\
\hline
\end{tabular}

Table 2. Beta Weights (Standardized) of the Regression Model with Post-treatment Backward Digit Span Score. The results indicate a significant effect for stimulation due to greater increase in the backward digit span score for transcranial random noise stimulation in comparison to transcranial direct current stimulation. $\mathrm{Std}=$ standard; $\mathrm{DF}=$ degrees of freedom

\begin{tabular}{lccccc} 
& $\beta$ & Std Error & DF & t-value & p-value \\
\hline Intercept & -0.197 & 0.108 & 51 & -1.811 & 0.076 \\
Stimulation & 0.331 & 0.156 & 51 & 2.123 & 0.038 \\
Backward digit span (baseline) & 0.84 & 0.077 & 51 & 10.928 & $<0.001$ \\
Time & 0.052 & 0.077 & 51 & 0.673 & 0.504 \\
\hline
\end{tabular}




\section{Supplemental Information to}

\section{Scaffolding the Attention-Deficit/Hyperactivity Disorder Brain Using Transcranial Direct}

\section{Current and Random Noise Stimulation: A Randomized Controlled Trial}

\section{Including}

Four Supplementary Figures

Nine Supplementary Tables

One Supplementary Methods

Supplementary Figure S1. Individual Attention-deficit/hyperactivity-disorder rating scale (ADHD-RS) scores at the baseline, after five days of the training, and one week after the interventions for transcranial random noise stimulation (tRNS) and transcranial direct current stimulation (tDCS). The order of each bar for each participant represent the score at baseline, after five days of the intervention, and one week after the intervention. Missing bars indicate a score of zero. Missing data were estimated using the regression equation and are represented by striped bars.

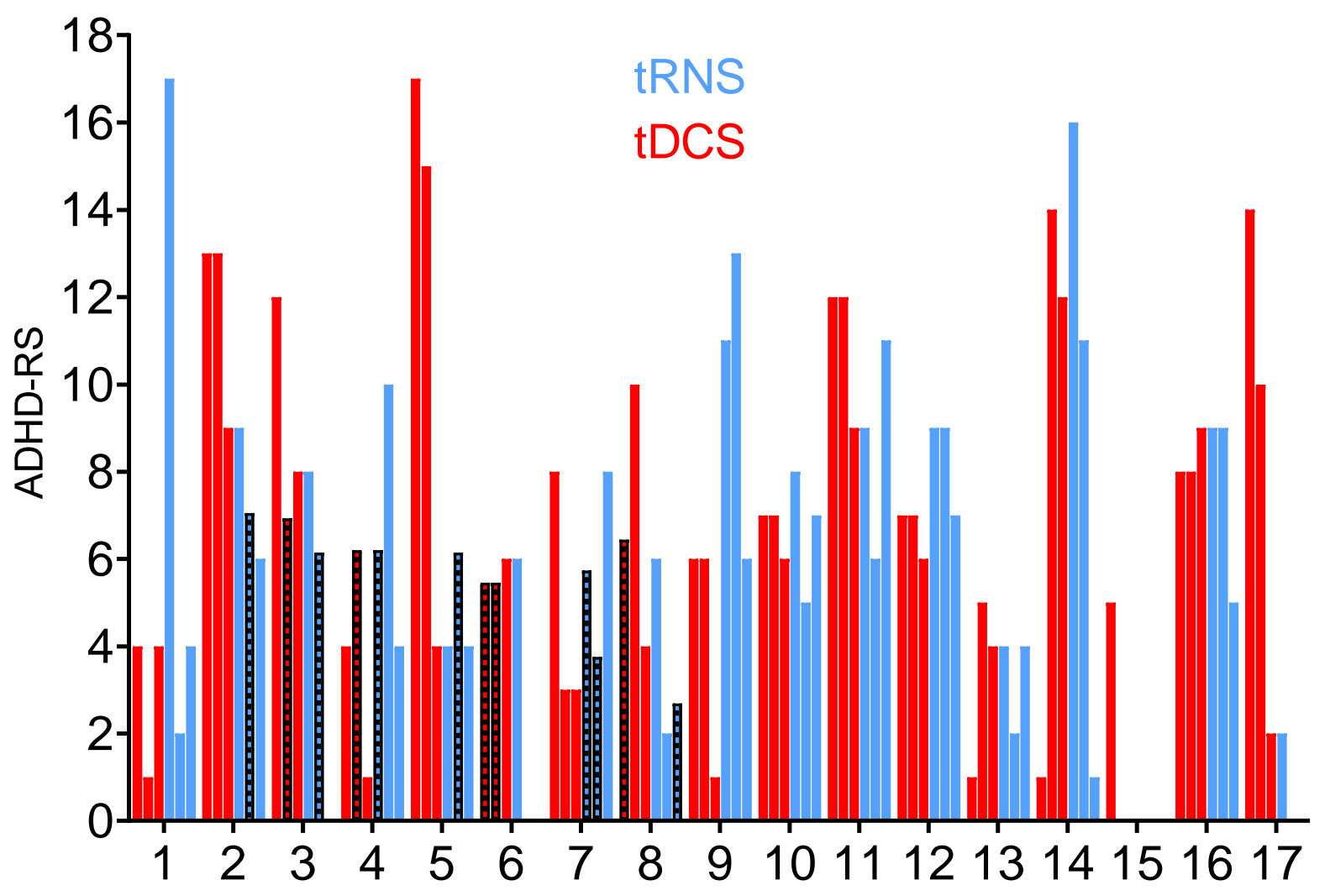


Supplementary Figure S2. Individual Attention-deficit/hyperactivity-disorder rating scale (ADHD-RS) improvement scores (baseline minus after the mean of five days of the training and one week after the interventions) for transcranial random noise stimulation (tRNS) and transcranial direct current stimulation (tDCS). Missing bars indicate a score of zero. Missing data were estimated using the regression equation.

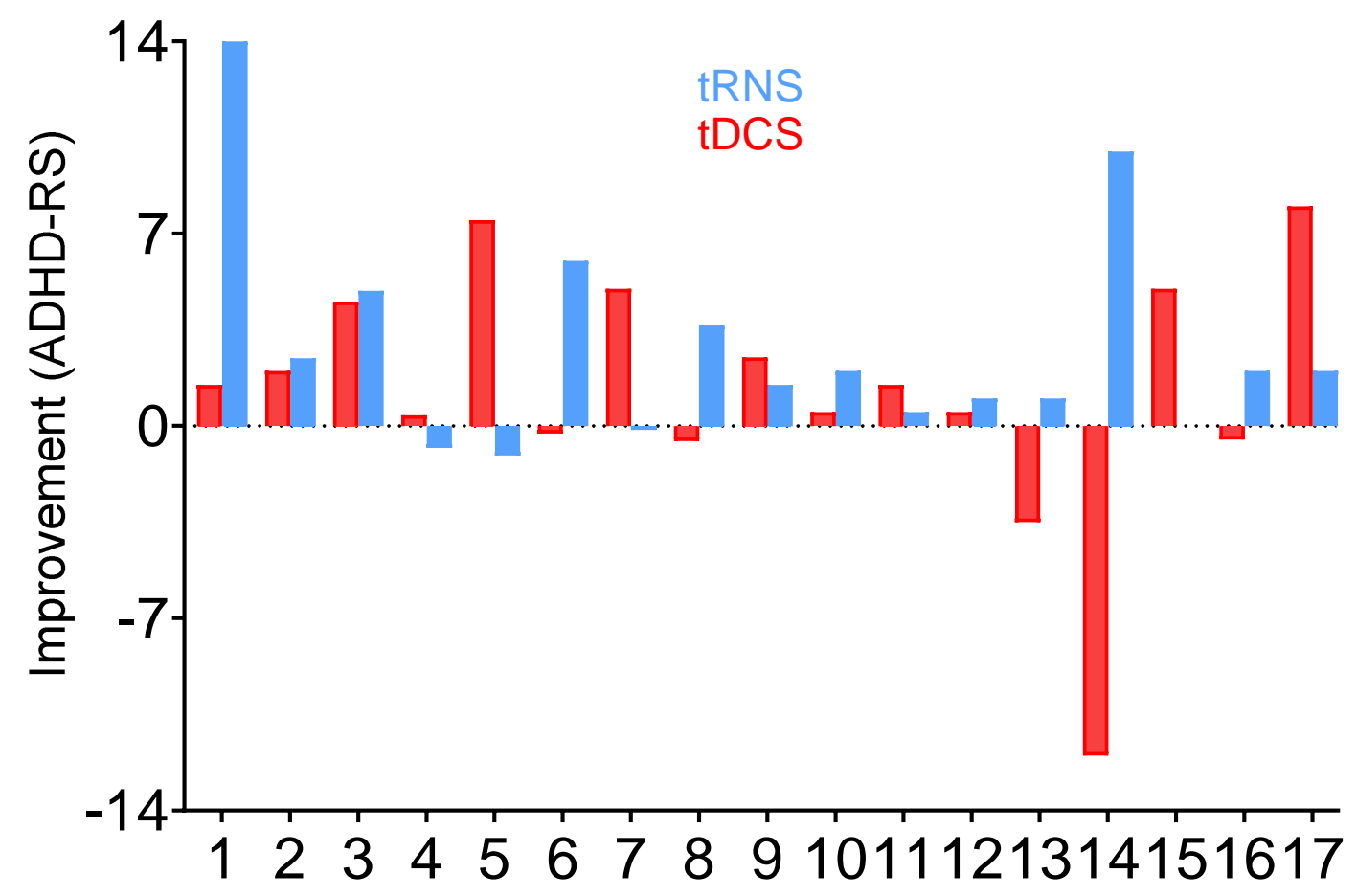

Patient \# 
Figure S3. Moderation model showing whether the improvement in the Attentiondeficit/hyperactivity-disorder rating scale (ADHD-RS) score as a function of stimulation (transcranial random noise stimulation vs. transcranial direct current stimulation) depends on the changes in the backward digit span score.

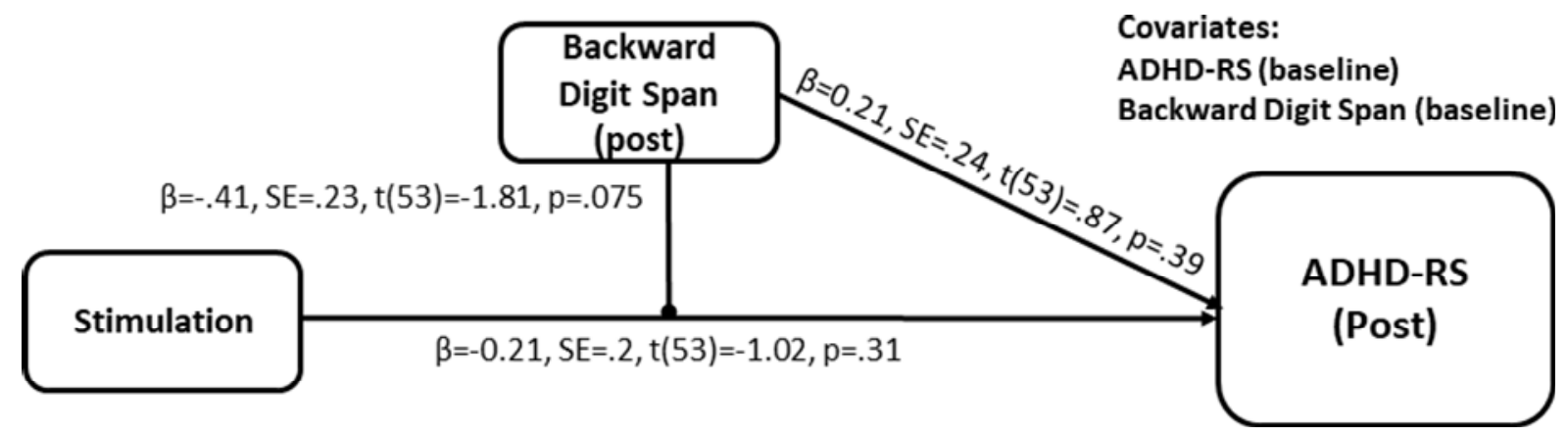


Figure S4. Computerized executive functions (EF) training by ACTIVATE ${ }^{\mathrm{TM}}$. Training comprised of four EF training exercises by ACTIVATE ${ }^{\mathrm{TM}}$, collectively targeting the EF functions of sustained attention, response inhibition, spatial working memory, cognitive flexibility and multiple simultaneous attention, which is the ability to switch attention between two or more tasks. Each training has 80-150 difficulty levels, the beginning sessions are short and easy to master, in order to accommodate a wide range of user ability and enable children to become familiar with the procedures. The difficulty level is adjusted up or down every 10-15 seconds, according to the child's abilities in order to optimize training effects. The computerized EF training use patented algorithms to individualize moment-to-moment difficulty and progression through different task configuration. The figure is presented with kind permission of Prof. Bruce Wexler, the developer of ACTIVATE ${ }^{\mathrm{TM}}$

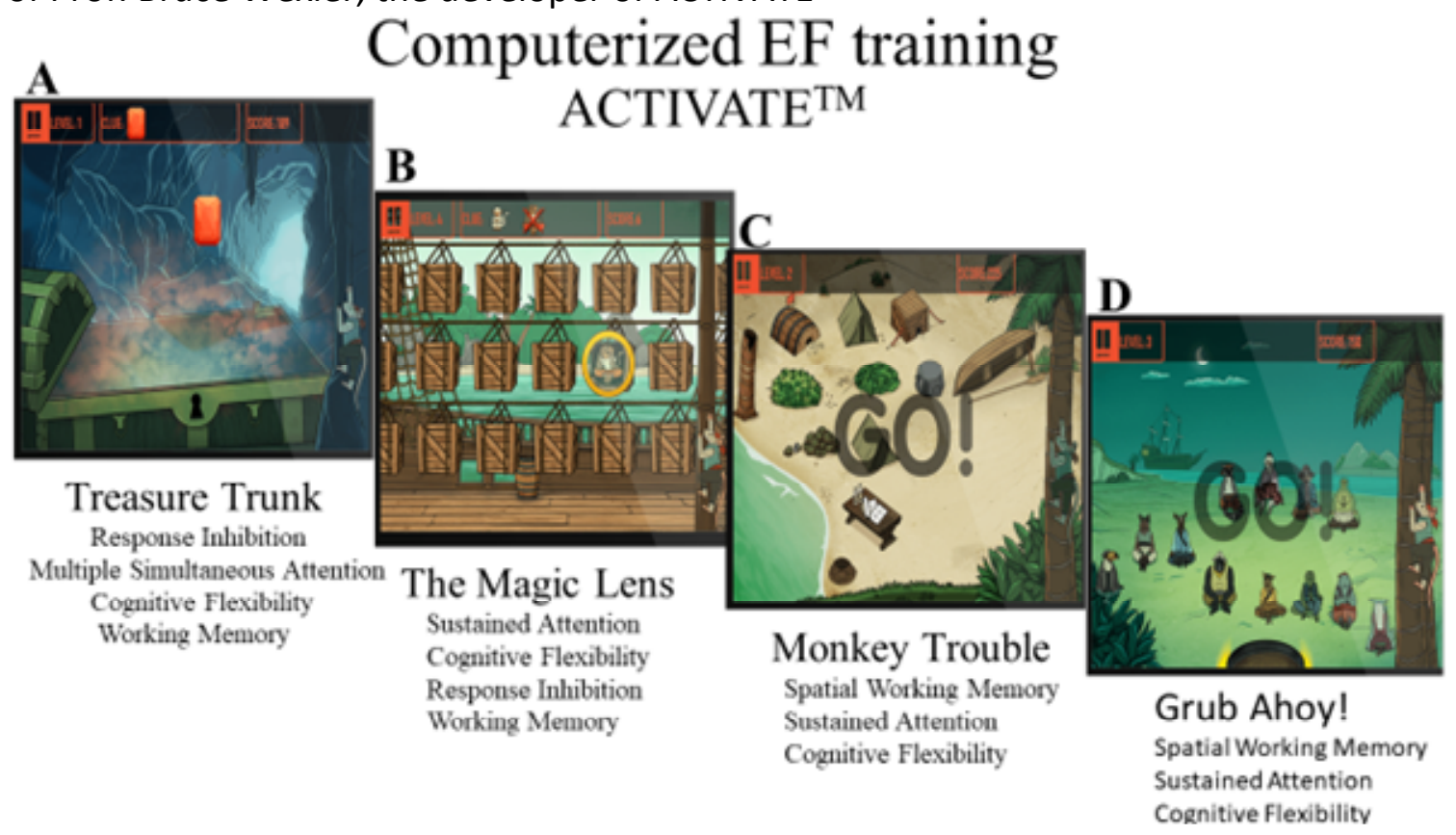


Table S1. Demographic data

\begin{tabular}{|c|c|c|c|c|c|}
\hline $\begin{array}{c}\text { Subject } \\
\#\end{array}$ & $\begin{array}{l}\text { Age } \\
\text { (year) }\end{array}$ & Gender & $\begin{array}{c}\text { ADHD } \\
\text { Subtype }\end{array}$ & $\begin{array}{l}\text { ADHD scores } \\
\text { before the start of } \\
\text { the intervention }\end{array}$ & $\begin{array}{c}\text { Baseline ADHD } \\
\text { scores }\end{array}$ \\
\hline 1 & $11: 05$ & $F$ & Type 3 & $\mathrm{I}=9, \mathrm{H}=8$ & 17 \\
\hline 2 & $11: 04$ & $M$ & Type 3 & $\mathrm{I}=8, \mathrm{H}=5$ & 13 \\
\hline 3 & 7:07 & $M$ & Type 2 & $\mathrm{I}=8, \mathrm{H}=9$ & 17 \\
\hline 4 & $6: 06$ & $M$ & Type 1 & & \\
\hline 5 & $6: 07$ & $M$ & Type 3 & $\mathrm{I}=5, \mathrm{H}=7$ & 12 \\
\hline 6 & 9:04 & M & Type 2 & & \\
\hline 7 & 7:02 & $M$ & Type 3 & $\mathrm{I}=8, \mathrm{H}=9$ & 17 \\
\hline 8 & $11: 04$ & $M$ & Type 1 & & \\
\hline 9 & $11: 04$ & $M$ & Type 1 & & \\
\hline 10 & 7:00 & $M$ & Type 3 & $\mathrm{I}=4, \mathrm{H}=2$ & 6 \\
\hline 11 & 9:07 & $M$ & Type 3 & $\mathrm{I}=5, \mathrm{H}=6$ & 11 \\
\hline 12 & $10: 02$ & $M$ & Type 2 & $\mathrm{I}=5, \mathrm{H}=3$ & 8 \\
\hline 13 & 9:11 & $M$ & Type 1 & $\mathrm{I}=8, \mathrm{H}=4$ & 12 \\
\hline 14 & $6: 05$ & $M$ & Type 1 & $\mathrm{I}=8, \mathrm{H}=1$ & 9 \\
\hline 15 & 9:08 & M & Type 1 & $\mathrm{I}=1, \mathrm{H}=0$ & 1 \\
\hline 16 & $10: 05$ & $M$ & Type 3 & $\mathrm{I}=7, \mathrm{H}=9$ & 16 \\
\hline 17 & $11: 09$ & M & Type 2 & $\mathrm{I}=3, \mathrm{H}=2$ & 5 \\
\hline 18 & $11: 06$ & $M$ & Type 2 & $\mathrm{I}=4, \mathrm{H}=4$ & 8 \\
\hline 19 & $8: 05$ & $M$ & Type 3 & $\mathrm{I}=9, \mathrm{H}=5$ & 14 \\
\hline
\end{tabular}

M: Male; F: Female; I: Inattentiveness; H: Hyperactivity/Impulsivity. ADHD: Attentiondeficit/hyperactivity-disorder. Note that all the children were unmedicated. Type $1=$ predominantly inattentive presentation, Type 2 = predominantly Hyperactive/Impulsive presentation, Type 3 = Combine presentation. The subtype diagnosis is based on clinical impression by the neurologist, using a detailed history, physical examination and ADHD-RS results. 
Table S2. Spontaneously Reported or Observed Adverse Events during transcranial direct current stimulation (tDCS) and transcranial random noise stimulation (tRNS). The table indicates the number of participants $(\mathrm{N})$ and the percentage of sessions endorsing side effects at some point during the intervention.

\begin{tabular}{lcccc} 
& \multicolumn{2}{c}{ tDCS $(\mathrm{n}=19)$} & \multicolumn{2}{c}{ tRNS $(\mathrm{n}=19)$} \\
\hline & $\mathrm{N}$ & $\%$ Sessions & $\mathrm{N}$ & \% Sessions \\
\hline Headache & 2 & 4 & & \\
Tingling & 2 & 6 & 2 & 5 \\
Itching & 6 & 13 & 4 & 7 \\
Local redness & 1 & 3 & & \\
Discomfort & 2 & 2 & & \\
\hline
\end{tabular}

Table S3. Beta Weights (Standardized) of the Regression Model with Post-treatment MOXO Attention Score as the Outcome Measure. Std= standard; DF= degrees of freedom.

\begin{tabular}{lccccc} 
& $\beta$ & Std.Error & DF & t-value & $p$-value \\
\hline (Intercept) & -0.1335 & 0.0917 & 51 & -1.4563 & 0.1514 \\
MOXO Attention (Baseline) & 0.0903 & 0.0381 & 51 & 2.3684 & 0.0217 \\
Stimulation & -0.0088 & 0.0258 & 51 & -0.3431 & 0.7329 \\
Time & 0.0344 & 0.0257 & 51 & 1.3357 & 0.1875 \\
\hline
\end{tabular}

Table S4. Beta Weights (Standardized) of the Regression Model with Post-treatment MOXO Impulsivity Score as the Outcome Measure. Std= standard; DF= degrees of freedom.

\begin{tabular}{lccccc} 
& $\beta$ & Std.Error & DF & t-value & $p$-value \\
\hline (Intercept) & -0.8218 & 0.0305 & 51 & -26.936 & $<0.0001$ \\
MOXO Impulsivity (Baseline) & -0.0631 & 0.0273 & 51 & -2.306 & 0.0252 \\
Stimulation & -0.0075 & 0.0241 & 51 & -0.3136 & 0.7551 \\
Time & -0.0043 & 0.0238 & 51 & -0.1833 & 0.8553 \\
\hline
\end{tabular}

Table S5. Beta Weights (Standardized) of the Regression Model with Post-treatment MOXO Hyperactivity Score as the Outcome Measure. Std= standard; DF= degrees of freedom.

\begin{tabular}{lccccc} 
& $\beta$ & Std.Error & DF & t-value & p-value \\
\hline (Intercept) & -0.1453 & 0.0393 & 51 & -3.692 & 0.0005 \\
MOXO Hyperactivity (Baseline) & -0.1414 & 0.0337 & 51 & -4.194 & 0.0001 \\
Stimulation & -0.0217 & 0.0279 & 51 & -0.777 & 0.4404 \\
Time & 0.0154 & 0.0281 & 51 & 0.549 & 0.5852 \\
\hline
\end{tabular}


Table S6. Beta Weights (Standardized) of the Regression Model with Post-treatment MOXO Timing Score as the Outcome Measure. Std= standard; DF= degrees of freedom.

\begin{tabular}{lccccc} 
& $\beta$ & Std.Error & DF & t-value & $p$-value \\
\hline (Intercept) & -0.235 & 0.256 & 47 & -0.915 & 0.364 \\
MOXO Timing (Baseline) & -0.115 & 0.105 & 47 & -1.095 & 0.279 \\
Stimulation & 0.249 & 0.12 & 47 & 2.068 & 0.044 \\
Time & -0.065 & 0.06 & 47 & -1.073 & 0.289 \\
\hline
\end{tabular}

Table S7. Beta Weights (Standardized) of the Regression Model with Post-treatment CGI-S Score as the Outcome Measure. Std= standard; DF= degrees of freedom.

\begin{tabular}{lccccc} 
& $\beta$ & Std.Error & DF & t-value & p-value \\
\hline (Intercept) & -0.333 & 0.113 & 53 & -2.955 & 0.005 \\
Stimulation & -0.067 & 0.09 & 53 & -0.753 & 0.455 \\
CGI-S (Baseline) & 0.082 & 0.093 & 53 & 0.881 & 0.383 \\
Time & -0.052 & 0.089 & 53 & -0.588 & 0.559 \\
\hline
\end{tabular}

Table S8. Beta Weights (Standardized) of the Regression Model with Post-treatment Forward Digit Span Score as the Outcome Measure. Std= standard; DF= degrees of freedom.

\begin{tabular}{lccccc} 
& $\beta$ & Std.Error & DF & t-value & $p$-value \\
\hline (Intercept) & -0.038 & 0.167 & 51 & -0.225 & 0.823 \\
Stimulation & 0.039 & 0.159 & 51 & 0.242 & 0.81 \\
Forward digit span (Baseline) & 0.381 & 0.114 & 51 & 3.328 & 0.002 \\
Time & 0.006 & 0.079 & 51 & 0.698 & 0.945 \\
\hline
\end{tabular}

Table S9. Moderation Analysis Predicting Post-treatment Attention-deficit/hyperactivitydisorder rating scale (ADHD-RS) score. The results revealed a trend toward significant interaction between stimulation type and post-treatment (post) backward digit span in predicting the post-treatment ADHD-RS score. Std= standard; DF= degrees of freedom.

\begin{tabular}{lccccc} 
& $\beta$ & Std Error & DF & t-value & p-value \\
\hline Intercept & 0.044 & 0.149 & 53 & 0.294 & 0.769 \\
ADHD-RS (baseline) & 0.638 & 0.117 & 53 & 5.456 & $<0.001$ \\
Stimulation & -0.208 & 0.205 & 53 & -1.018 & 0.313 \\
Backward Digit Span (post) & 0.209 & 0.241 & 53 & 0.868 & 0.389 \\
Backward Digit Span (baseline) & 0.008 & 0.192 & 53 & 0.042 & 0.966 \\
Stimulation*Backward Digit Span (post) & -0.412 & 0.227 & 53 & -1.813 & 0.0754 \\
\hline
\end{tabular}




\section{Supplementary Methods}

\section{A description of the Computerized executive functions (EF) training (ACTIVATE ${ }^{\text {TM}}$ )}

The computerized training used in the study (ACTIVATE ${ }^{\text {TM }}$ by $c 8$ sciences,

https://www.c8sciences.com/) is comprised of four EF training games, collectively targeting the $\mathrm{EF}$ functions of sustained attention, response inhibition, spatial working memory, cognitive flexibility, switching, and divided attention. The code for the games was created with the text editor Sublime (https://www.sublimetext.com/) and artwork created in Photoshop (http://www.photoshop.com/).

The EF training starts after introducing to the child a Pirate island and asking him to choose a game. There are two versions of two training games with the same underlying computer code and sequence of cognitive challenges, but a different user game experience. While Treasure trunk and Magic lens target mainly response inhibition, Monkey trouble and Grub Ahoy target mainly working memory (Figure S4). Exercises instructions are presented both aurally and visually on the screen. The beginning sessions are short and easy to master, the difficulty level is adjusted up or down every 10-15 seconds, according to the child's abilities. The computerized EF training use algorithms to individualize moment-to-moment difficulty and progression through different task configuration. The cognitive software recognizes a child's strengths and weaknesses and can detect when a child makes an error; when the child makes an error, the program coaches him until he successfully corrects the error and moves on to the next level; by giving auditory (special ding for correct answers and another ding for errors) and/or visual feedback (i.e., excellent, congratulations). Computer code for the games was created with the text editor Sublime (https://www.sublimetext.com/) and art work created in Photoshop (http://www.photoshop.com/).

A. Treasure Trunk trains simple sustained attention at its initial levels, but systematically adds discriminant attention, response inhibition, cognitive flexibility, working memory and multiple simultaneous attention to constitute a general executive function training. In this exercise, children begin by using the mouse to track a moving light and click on it when it turns into a red jewel. With correct responses, the light moves faster, with mistakes it slows down. As the exercise continues, more aspects of executive function are added. Blue jewels appear that should not be clicked, adding discriminant attention and response inhibition. Next, the target switches randomly between red and blue, increasing response inhibition demands and adding cognitive flexibility. Working memory is introduced by showing halfjewels and instructing children to only click on one that is the same color as the one before in order to create a full jewel. In addition to the primary focus on executive functions, this training also requires visual-spatial processing and hand-eye coordination. All levels repeat with two and three moving lights on the screen.

B. The Magic Lens. Targets sustained attention, cognitive flexibility, response inhibition and working memory. In this exercise the child must first find the monkeys hidden in the pirates' crates. When the magic lens reveals a monkey, the child should click the monkey to free it from the crate. The movement speed of the lens is adaptive and depends on the accuracy. In higher levels of difficulty, the child needs to search for other objects in the crates - first pirate monkeys and, later, boots, which is more difficult. The child uses clues at the top of the screen to know what objects they are to follow; which kind of monkey/boot he should free.

C. Monkey Trouble. In this exercise, the child sees a pattern of objects appear on screen for a short period of time. When the pattern disappears, the child has to recreate it by clicking on the specific objects in the correct order. The pattern gets longer by 1 item with every 
several correct answers, and shorter if the child makes a mistake. As the levels of the exercise increase, the child will need to be able to match the correct pattern in reverse.

D. Grub Ahoy!. In this exercise, the child sees a pattern of pirates appear on screen, created by hungry pirates. Children have to remember the order in which a group of pirates seated on the beach raise their hands to request dinner, or the places in a campsite visited by a playful monkey, clicking on them in appropriate order. The number of locations to be remembered begins with two, increases with success and decreases with mistakes. Some levels require the child to respond in reverse order. 